\title{
ON NONISOMORPHIC ANALYTIC SETS
}

\author{
R. DANIEL MAULDIN
}

\begin{abstract}
It is shown that if $A$ is an analytic subset of $I$, the unit interval, such that $I-A$ is uncountable and does not contain a perfect set, then $A$ is not Borel isomorphic to $I \times A$ or to $A^{n}, n>1$, or to $U$, where $U$ is a universal analytic subset of $I^{2}$. It is also shown that $U$ is not isomorphic to $I \times A$ or to $A^{n}, n>1$.
\end{abstract}

We shall say that two Borel structures (or measurable spaces) $(X, \Sigma)$ and $(Y, \mathbb{Q})$ are isomorphic provided there is a one-to-one measurable map of $X$ onto $Y$ whose inverse is also measurable. It can be seen that if $X$ is isomorphic to a subset $Z$ of $Y$, where $Z \in \mathbb{Q}$, and $Z$ has the restricted Borel structure, and $Y$ is isomorphic to a subset $E$ of $X$, where $E \in \Sigma$ and $E$ has the restricted Borel structure, then $(X, \Sigma)$ and $(Y, \mathscr{Q})$ are isomorphic. It is well known that if $B_{1}$ and $B_{2}$ are Borel subsets of Polish spaces provided with the relative Borel structure, then $B_{1}$ and $B_{2}$ are isomorphic if and only if they have the same cardinality.

The problem of the number of isomorphism classes of analytic, nonborelian subsets of Polish spaces seems to be unsolved. In [1], A. Maitra and C. RyllNardzewski show that (i) any two universal analytic sets are isomorphic, and (ii) if $A$ is an analytic set whose complement is uncountable and does not contain a perfect set, then $A$ is not in the isomorphic class of the universal analytic sets.

In this note we give some corollaries of the techniques employed in [1], and recount their main argument in Theorem 1.

First, let us set some notation. The unit interval will be denoted by $I$ and $A$ will denote an analytic subset of $I$ whose complement is uncountable and does not contain a perfect set. The existence of such a set is implied by Gödel's Axiom of Constructibility [2]. The $n$-fold product of $A$ with itself is denoted by $A^{n}$. The symbol $U$ will denote a universal analytic subset of $I \times I$. The dyadic rationals are denoted by $R_{0}$. The binary sieve of Lebesgue is used throughout this paper [3, p. 34].

In this note, we show that no two of the following sets are isomorphic: $A, I \times A$, and $U$. We show that $U$ and $A^{n}, n \geqslant 1$, are not isomorphic. Also, $A$

Received by the editors August 26, 1975 and, in revised form, November 10, 1975.

AMS (MOS) subject classifications (1970). Primary 02K30, 54H05; Secondary 28A05.

Key words and phrases. Analytic sets, sieves, universal sets, Borel isomorphism, Axiom of Constructibility.

(1) American Mathematical Society 1976 
and $A^{n}, n>1$, are shown to be nonisomorphic.

Results.

THEOREM 1. If $B$ is an analytic subset of a Polish space $S$ and uncountably many of the constituents of $B$ with respect to some sieve are uncountable, then $B$ is not isomorphic to $A$.

Proof. Let $\left\{C_{\alpha}\right\}_{\alpha<\omega_{1}}$ be the constituents of $S-B$ [3, p. 499] with respect to some sieve so that uncountably many of the $C_{\alpha}$ 's are uncountable.

Now, suppose $\phi$ is a Borel isomorphism of $A$ onto $B$. We may suppose $Z_{1}$ is a Borel subset of $I, Z_{1} \supseteq A, Z_{2}$ is a Borel subset of $S, Z_{2} \supseteq B$ and $\phi$ has been extended to a Borel isomorphism of $Z_{1}$ onto $Z_{2}$ [3, p. 436].

There is an ordinal $\alpha_{0}, \alpha_{0}<\omega_{1}$, such that $S-Z_{2} \subseteq \cup_{\beta<\alpha_{0}} C_{\beta}$. Fix $\alpha_{1} \geqslant \alpha_{0}$ such that $C_{\alpha_{1}}$ is uncountable. Then $C_{\alpha_{1}} \subseteq Z_{2}-B$ and $\phi^{-1}\left(C_{\alpha_{1}}\right)$ is an uncountable Borel set lying in $Z_{1}-A$. Contradiction.

Corollary 1. The set $A$ is not isomorphic to $I \times A$.

Proof. Let $L$ be a sieve of Borel sets such that $A$ is the set sifted by $L$. Let $\left\{C_{\alpha}\right\}_{\alpha<\omega_{1}}$ be the constituents of $A^{\prime}$ with respect to the sieve $L$. It is easy to show that $\left\{I \times C_{\alpha}\right\}_{\alpha<\omega_{1}}$ are the constituents of $I \times A^{\prime}$ with respect to the sieve $L_{1}$, where $L_{1}$ is the map from $R_{0}$ into the Borel subsets of $I \times I$, defined by $L_{1}(r)=I \times L(r)$. The corollary follows from Theorem 1 .

Corollary 2. The set $A$ is not isomorphic to $A \times A$.

Proof. Let $L$ be a sieve of closed subsets of $I \times I$ which sifts $A \times A[3, \mathrm{p}$. 483]. Suppose there is an ordinal $\alpha_{0}$ such that if $\alpha \geqslant \alpha_{0}, C_{\alpha}$ is countable, where $\left\{C_{\alpha}\right\}_{\alpha<\omega_{1}}$ are the constituents of $I^{2}-A^{2}$ with respect to $L$.

Then $B=\cup_{\alpha \leqslant \alpha_{0}} C_{\alpha}$ is a Borel subset of $I \times I$ and $B \subseteq(I \times I)-(A \times A)$. Thus, $\Pi_{2}(B \cap(A \times I))$, the projection of $B \cap(A \times I)$ into the second axis, is an analytic subset of $I$ disjoint from $A$. Thus, $D_{2}=\Pi_{2}(B \cap(A \times I))$ is countable. Similarly, $D_{1}=\Pi_{1}(B \cap(I \times A))$ is countable.

Thus, $K=B-\left[\left(D_{1} \times I\right) \cup\left(I \times D_{2}\right)\right]$ is a Borel set lying in $A^{\prime} \times A^{\prime}$. From this it follows that $E=\Pi_{1}(K)$ is countable.

Let $x \in I-\left(A \cup D_{1} \cup E\right)$. Then $\{x\} \times\left(I-D_{2}\right)$ is a Borel set lying in $(I \times I)-[(A \times A) \cup B]$.

For some $\gamma_{0}, \gamma_{0}<\omega_{1},\{x\} \times\left(I-D_{2}\right) \subset \cup_{\alpha_{0}<\alpha<\gamma_{0}} C_{\alpha}$. Thus, some $C_{\alpha}$, with $\alpha_{0}<\alpha<\gamma_{0}$ must be uncountable. Therefore, uncountably many of the constituents of $A \times A$ are uncountable and Corollary 2 now follows from Theorem 1. This answers a problem stated by S. Ulam on p. 10 of his problem book [4] assuming the existence of such a set as $A$. This type of problem is also raised by J.P.R. Christensen in his book [7, pp. 46-47] and answers some of the problems posed there by him.

We shall now demonstrate a property which the sets $A^{n}, n>1$, have with respect to any Polish space in which they are embedded, which prevents them from being isomorphic to $U$. 
THEOREM 2. For each $n>1$, there is a family $\mathcal{G}_{n}$ of $\aleph_{1}$ subsets of $I^{n}$ filling up $I^{n}-A^{n}$ such that (1) every analytic subset of $I^{n}-A^{n}$ is covered by a countable subfamily of $\mathcal{G}_{n}$ and (2) each set in $\mathcal{S}_{n}$ is an $F_{\sigma}$ set.

Proof. The proof proceeds by induction.

First, let us show this for $n=2$ : Again let $L$ be a sieve of Borel sets such that $A$ is the set sifted by $L$. Let $\left\{C_{\alpha}\right\}_{\alpha<\omega_{1}}$ be the constituents of $A^{\prime}$ with respect to the sieve $L$. The sets $C_{\alpha}$ are countable.

Let $\mathcal{G}_{2}=\left\{\left(C_{\alpha} \times I\right) \cup\left(I \times C_{\alpha}\right): \alpha<\omega_{1}\right\} ; \mathcal{G}_{2}$ is a family of $\boldsymbol{\aleph}_{1}$ subsets of $I^{2}-A^{2}$ and clearly each set in $\mathcal{G}$ is an $F_{\sigma}$ set. If $B$ is an analytic set sitting in $I^{2}-A^{2}$, then there is an ordinal $\alpha_{0}$ such that $D_{1} \cup D_{2} \subset \cup_{\beta<\alpha_{0}} C_{\beta}$, where $D_{1}=\Pi_{1}(B \cap(I \times A))$ and $D_{2}=\Pi_{2}(B \cap(A \times I))$. Thus, $K=B-\left[\left(D_{1}\right.\right.$ $\left.\times I) \cup\left(I \times D_{2}\right)\right]$ is an analytic set lying in $A^{\prime} \times A^{\prime}$. Therefore, there is an ordinal $\alpha_{1}, \alpha_{0}<\alpha_{1}<\omega_{1}$, so that $\Pi_{i}(K) \subset \cup_{\beta<\alpha_{1}} C_{\beta}, i=1$, 2. It follows that $B$ is covered by $\left\{\left(C_{\alpha} \times I\right) \cup\left(I \times C_{\alpha}\right): \alpha<\alpha_{1}\right\}$.

Now, let us suppose the existence of such a family $\mathcal{G}_{n}$ has been shown for the positive integer $n, n>1$.

Let $\mathcal{G}_{n+1}=\left\{\left(I^{n} \times C_{\alpha}\right) \cup(K \times I): \alpha<\omega_{1}\right.$ and $\left.K \in \mathcal{G}_{n}\right\}$. Clearly, $\left|\mathcal{S}_{n+1}\right|$ $=\aleph_{1}$ and each set in $\mathcal{G}_{n+1}$ is an $F_{\sigma}$ set. That $\mathcal{G}_{n+1}$ has the required covering property may be shown by an argument similar to that given for the case $n=2$.

Theorem 3. Suppose $D$ is an analytic subset of a Polish space $S$ such that there is a family of $G$ of $\aleph_{1}$ Borel subsets of $S$ filling up $S-D$ such that (1) if $E$ is an analytic subset of $S$ lying in $S-D$, then $E$ is covered by countably many members of $G$ and (2) there is an ordinal $\alpha, \alpha<\omega_{1}$, such that each member of $G$ is of Borel's additive class $\alpha$. Then $D$ is not isomorphic to $K$, if $K$ is an analytic subset of $I^{2}$ such that Borel sets of arbitrarily high class appear as vertical sections of $K$.

Proof. Suppose $D$ is isomorphic to $K$. We may assume that $Z_{1}$ is a Borel subset of $S$ with $Z_{1} \supset D, Z_{2}$ is a Borel subset of $I^{2}$ with $Z_{2} \supset K$ and $\phi$ is an isomorphism of $Z_{1}$ onto $Z_{2}$ which takes $D$ onto $K$. Let $\gamma$ be an ordinal such that if $E$ is of Borel's additive class $\alpha$ with respect to $Z_{1}$, then $\phi(E)$ is of additive class $\gamma$ in $I^{2}$. In particular, $Z_{2}$ is of additive class $\gamma$ in $I^{2}$ and if $H \in G, \phi(H)$ is of additive class $\gamma$ in $I^{2}$.

Let $x$ be a point of $\Pi_{1}(K)$ such that the fibre of $K$ over $x, K_{x}$, is a Borel subset of $I$, but not of additive class $\gamma+2$. Let $T=\left(Z_{2}\right)_{x}-K_{x}$. Let $\left\{H_{n}\right\}_{n=1}^{\infty}$ be a sequence of sets from $\mathcal{G}$ such that $\phi^{-1}(T) \subset \cup_{n=1}^{\infty} H_{n}$. Then $W$ $=\phi\left(\cup H_{n}\right)$ is of additive class $\gamma$ in $I^{2}$. Since $\left(I^{2}-Z_{2}\right)_{x}$ is of additive class $\gamma+1$ in $I, W_{x} \cup\left(I^{2}-Z_{2}\right)_{x}=I-K_{x}$ is of additive class $\gamma+1$ in $I$. Therefore, $K_{x}$ is of additive class $\gamma+2$ in $I$. This contradiction proves the theorem.

Corollary 3. For each $n, n>1$, the set $A^{n}$ is not isomorphic with $U$. Also, the sets $I \times A$ and $U$ are not isomorphic.

Proof. That $A^{n}$ and $U$ are not isomorphic follows immediately from 
Theorems 2 and 3 and the fact that all Borel subsets of $I$ appear as vertical sections of $U$. That $I \times A$ and $U$ are not isomorphic follows from Theorem 3 and the fact that there is a collection $G$ of $\aleph_{1}$ subsets of $I^{2}-(I \times A)$ satisfying the hypothesis of Theorem 3. (This is indicated in the proof of Corollary 1.)

Finally, let us note that the methods used in this note to show that two analytic sets are not isomorphic involve an analysis of the structure of the complements of these sets in any Polish space in which they are embedded. There are, however, two intrinsic invariants of the isomorphism classes of analytic sets. They are put forth in the following theorems.

THeOREM 4. The analytic sets $A_{1}$ and $A_{2}$ are isomorphic if and only if the Banach spaces consisting of the bounded Baire functions over each set provided with the least upper bound norm are isometrically isomorphic.

An argument for Theorem 5 is given in Theorem 2 of [5].

TheOrem 5. The analytic sets $A_{1}$ and $A_{2}$ are isomorphic if and only if the semigroups of analytic relations over these sets are isomorphic as semigroups.

This is a corollary of Theorem 2.2 of [6].

Finally, I would like to close with the

Question. If $n \neq m$, is it true that $A^{n}$ is not isomorphic to $A^{m}$ ?

\section{REFERENCES}

1. A. Maitra and C. Ryll-Nardzewski, On the existence of two analytic non-Borel sets which are not isomorphic, Bull. Acad. Polon. Sci. Sér. Sci. Math. Astronom. Phys. 18 (1970), 177-178. MR 42 \#3743.

2. K. Gödel, The consistency of the axiom of choice and of the generalized continuum-hypothesis, Proc. Nat. Acad. Sci. 24 (1938), 556-557.

3. C. Kuratowski, Topology. Vol. I, Academic Press, New York, 1966. MR 36 \# 840.

4. S. Ulam, Problems in modern mathematics, Wiley, New York, 1964. MR 43 \#6031.

5. John E. Jayne, The space of class $\alpha$ Baire functions, Bull. Amer. Math. Soc. 80 (1974), 1151-1156. MR 50 \# 14647.

6. K. D. Magill, Jr., Isomorphisms of triform semigroups, J. Austral. Math. Soc. 10 (1969), 185-193. MR 39 \# 7010.

7. J. P. R. Christensen, Topology and Borel structure, North-Holland, Amsterdam; American Elsevier, New York, 1974. MR 50 \# 1221. 